\title{
Factores de riesgo para la adquisición y características microbiológicas de las bacteriemias por Acinetobacter baumannii multi-resistente en pediatría. Estudio de casos y controles
}

\author{
Silvina Ruvinsky, Graciela Fiorilli, M. Guadalupe Pérez, Eduardo Motto, \\ Carlos Cambaceres, Leonardo Mannino, Horacio Lopardo y Rosa Bologna
}

Microbiological features and risk factors for acquiring multidrug-resistant Acinetobacter baumannii bacteremia in pediatric patients. Case-control study

Background: Multidrug-resistant Acinetobacter baumannii (MAB) is an important nosocomial pathogen. Objectives: To analyze the risk factors for acquiring MAB, and the clinical and microbiological characteristics of MAB bacteremia (MABB) in children. Materials and Methods: Control-case study 2005-2008. Demographic and clinical data from all $\mathrm{MABB}$ and from non-multiresistant gram-negative bacteremias were recorded. Identification at species level, antimicrobial susceptibility tests, time-kill studies and clonally relationships were performed. Stata 8.0 was used for data analysis. Results: A total of $50 \mathrm{MABB}$ and 100 controls were included. Ninety four percent of patients acquired MAB in ICU and the $88 \%$ had underlying diseases. All patients had invasive procedures previous to MABB. The median of hospitalization stay previous to MABB was different in cases than in controls (16 vs 7 days, $p<0.001$ ). Five clones were detected among the MABB. Time-killing curves showed bactericidal activity of ampicillin/sulbactam plus gentamicin and polymixin B. Three patients with MAB died. In a multivariate analysis final predictors of MABB were: previous use of broad-spectrum antibiotics [OR: 7,0; IC 95\% 1,93-25,0; p: 0,003] and mechanical ventilation [OR: 4,19; IC 95\% 1,66-10,0; p: 0,002]. Conclusions: MABB were detected in patients with underlying conditions, invasive procedures and prolonged hospitalization. Predictors of MABB were mechanical previous use of broad-spectrum antibiotics and mechanical ventilation.

Key words: Acinetobacter baumannii, multidrug-resistance, colistin, pediatric.

Palabras clave: Acinetobacter baumannii, multi-resistencia, colistina, pediatría.

\section{Introducción}

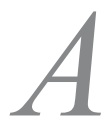
cinetobacter baumannii multi-resistente (ABM), se ha convertido, en estos últimos años en un patógeno de importancia en el ámbito hospitalario ${ }^{1}$. Es un microorganismo oportunista, capaz de sobrevivir por largos períodos en superficies y causar infecciones en pacientes que requieren internación en las unidades de cuidados intensivos (UCI). Es causa frecuente de infecciones asociadas al cuidado de la salud: neumonía asociada al ventilador, bacteriemia relacionada a catéter venoso central, meningitis asociada a shunt $\mathrm{e}$ infecciones del tracto urinario.

Las infecciones por ABM tienen impacto en el sistema de salud no sólo por su morbi-mortalidad asociada sino porque prolongan la internación y producen un incremento en los costos ${ }^{2}$.

Son escasos los estudios poblacionales sobre las infecciones producidas por $\mathrm{ABM}$ en niños ${ }^{3}$.
El objetivo del estudio fue analizar características clínicas, epidemiológicas, microbiológicas y factores de riesgo para su adquisición en los pacientes con bacteriemias por $\mathrm{ABM}$ en comparación con otros bacilos gramnegativos en un hospital pediátrico.

\section{Material y Métodos}

El estudio se efectuó en el Hospital de Pediatría "Prof. Dr. Juan P. Garrahan" de la ciudad de Buenos Aires, Argentina. Es un hospital pediátrico de alta complejidad que posee una capacidad de 620 camas, incluidas dos UCI polivalentes, una de quemados y otra de cuidados intensivos cardiovasculares.

Se realizó un estudio retrospectivo de casos y controles de pacientes con bacteriemia por ABM internados en las UCI del hospital, en el período comprendido entre el $1^{\circ}$ de enero de 2005 y el 31 de diciembre de 2008 . Se
Ciudad de Buenos Aires. Argentina.

Hospital de Pediatría Juan. P. Garrahan.

Servicio de Control Epidemiológico e Infectología (SR, MGP, LM, RB). Servicio de Microbiología (GF, HL). Unidad de Cuidados intensivos (EM, CC).

Los autores no presentan conflictos de interés.

El trabajo fue parcialmente

financiado mediante un subsidio

Roemmers para investigación en Epidemiología.

Recibido: 25 de abril de 2014 Aceptado: 30 de octubre de 2014

\section{Correspondencia a:}

Silvina Ruvinsky sruvinsky@hotmail.com 
incluyeron pacientes de un mes a 16 años con al menos $48 \mathrm{~h}$ de internación en UCI, con aislamiento de ABM en hemocultivos considerados como clínicamente relevantes (casos) y pacientes con bacteriemias por otros bacilos gramnegativos (controles) internados durante el mismo período en las UCI. La selección de casos y controles se realizó al azar en base a los registros electrónicos del Servicio de Microbiología considerando una relación de dos controles por cada caso incluido.

\section{Análisis clínico y epidemiológico}

Mediante revisión de historias clínicas se registraron variables demográficas, enfermedad de base y procedimientos invasores (catéter venoso central-CVC, catéter vesical, asistencia respiratoria mecánica $[\mathrm{ARM}]$ o cirugía) previo a la aparición de la bacteriemia. Asimismo se consideró el uso previo de antimicrobianos, tiempo de estadía en UCI y la evolución final de los pacientes ingresados al estudio. Para la clasificación de las bacteriemias se utilizaron los criterios definidos por los Centers for Disease Control and Prevention (CDC) de Atlanta, E.U.A. ${ }^{4}$. En los pacientes con $\mathrm{CVC}$ con 48 h o más de permanencia, se consideró bacteriemia asociada a CVC ante ausencia de foco clínico de infección y uno más de los siguientes criterios: fiebre mayor a $38^{\circ} \mathrm{C}$, escalofríos o hipotensión arterial y confirmación microbiológica mediante aislamiento de un patógeno en uno o más hemocultivos. Se consideró bacteriemia primaria si se documentaron hemocultivos positivos sin foco clínico de infección, urocultivo negativo $\mathrm{y}$ en pacientes $\sin \mathrm{CVC}$ en ese momento.

\section{Estudios microbiológicos}

Se efectuó la identificación de la especie de Acinetobacter mediante pruebas fenotípicas convencionales como las recomendadas por Bouvet y Grimont ${ }^{5}$, como pruebas genotípicas que incluyeron la hibridación con una sonda específica para el gen oxa51 (dot blot), y posterior secuenciación ${ }^{6}$. Los resultados dudosos y los negativos se confirmaron con reacción de polimerasa en cadena (RPC) específica para el mismo gen y por secuenciación parcial del gen ADNr 16S amplificado con los cebadores universales $27 \mathrm{~F}$ y $1492 \mathrm{R}$.

Los estudios de la susceptibilidad antimicrobiana por el método de difusión se realizaron según las recomendaciones del CLSI para los siguientes antimicrobianos: piperacilina (PIP), ampicilina/sulbactam (AMS), piperacilina/ tazobactam (TAZ), amoxicilina/ácido clavulánico (AMC), ceftazidima (CAZ), cefepima (FEP), meropenem solo (MEM) o con EDTA, imipenem solo (IMI) o con EDTA, colistín (COL), amikacina (AK), gentamicina (GEN), ácido nalidíxico (NAL), ciprofloxacina (CIP), cotrimoxazol (STX) y minociclina (MIN) ${ }^{7}$. Por el método de dilución se ensayaron MEM, MEM + EDTA, IMI e IMI + EDTA. Se realizó búsqueda de $\beta$-lactamasas de espectro extendido
$(B L E E)^{8}$ y de carbapenemasas (metalo- $\beta$-lactamasas y oxacilinasas) por métodos fenotípicos ${ }^{9}$ y genotípicos ${ }^{10}$. Se estudió la presencia de otras oxacilinasas con actividad frente a los carbapenemes (OXA 23 y OXA 58) por el mismo método de dot blot. Los resultados dudosos se confirmaron por RPC.

Se efectuaron estudios de clonalidad de los aislados por RPC con oligonucleótidos degenerados (sigla en inglés DO-PCR) ${ }^{11}$.

A los aislados representantes de cada clon se les probó AMS $(16 / 8 \mu \mathrm{g} / \mathrm{ml}$ ), polimixina B (POL $0,25 \mu \mathrm{g} / \mathrm{ml}$ ) y la combinación AMS + GEN $(16 / 8$ y $4 \mu \mathrm{g} / \mathrm{ml}$ respectivamente) por curva de letalidad. Se trabajó con concentraciones factibles de ser alcanzadas en suero, según datos tomados de Lorian $^{12}$.

- Concentraciones bacterianas de alrededor de $10^{6} \mathrm{ufc} /$ $\mathrm{ml}$ se enfrentaron con los antimicrobianos solos o en combinación y fueron incubadas a $35^{\circ} \mathrm{C}$. Se tomaron alícuotas de las suspensiones a las 0,4 y 24 h o a las 0 , 3, 6 y 24 h de incubación, según el caso. Por dispersión de las gotas en medio sólido se descartó la posibilidad de existencia de efecto carry-over.

Polimixina B, se utilizó en lugar de colistín en los ensayos in vitro, dado que los resultados obtenidos con ambos antimicrobianos son homologables ${ }^{13}$.

\section{Análisis estadístico}

Se estimó media, mediana, rango intercuartilo (RIC) para las variables continuas. Análisis de variables continuas: t-test o test de Wilcoxon según correspondiese. Variables categóricas: $\chi^{2}$ o test de Fisher dependiendo del tamaño de muestra. Se realizó análisis univariado y regresión logística. Luego de explorar por separado cada variable independiente (edad, sexo, enfermedad de base, tipo de infección, ARM, CVC, catéter vesical, cirugía previa) y variable dependiente categórica (bacteriemia por $\mathrm{ABM}$ ), se seleccionaron las mejores variables tomando en cuenta los confundidores, posibles interacciones (selección forward realizada en forma manual). Luego se tuvo en cuenta la adecuación del modelo (Hosmer $\&$ Lemeshow). El valor de $\mathrm{p}<0,05$ fue definido como estadísticamente significativo. Para el análisis se utilizó STATA 8.0.

\section{Resultados}

Se evaluaron 50 pacientes con bacteriemia por ABM (casos) y un total de 100 pacientes con bacteriemias por otros bacilos gramnegativos (controles) adquiridas en las UCI.

Los 50 aislados de ABM fueron confirmados como pertenecientes a la especie $A$. baumannii. 
Tabla 1. Características generales de los pacientes con bacteriemias por Acinetobacter baumannii multi-resistentes y de sus controles

\begin{tabular}{|c|c|c|c|c|c|c|c|c|}
\hline \multirow{2}{*}{\multicolumn{2}{|c|}{ Variable }} & \multicolumn{2}{|c|}{ Casos (n: 50) } & \multicolumn{2}{|c|}{ Controles $^{\vee}(n: 100)$} & \multicolumn{2}{|c|}{ OR (IC 95\%) } & \multirow[t]{2}{*}{ p } \\
\hline & & $\mathrm{n}$ & (\%) & & (\%) & & & \\
\hline \multicolumn{2}{|l|}{ Sexo masculino } & 29 & (58) & 51 & (51) & 1,33 & $(0,6-2,63)$ & 0,41 \\
\hline \multicolumn{2}{|l|}{ Edad (meses)* } & 13,5 & $(6-54)$ & 23 & $(4-81)$ & & & 0,63 \\
\hline \multicolumn{2}{|l|}{ Enfermedad de base } & 45 & (90) & 86 & (86) & 1,46 & $(0,5-4,32)$ & 0,48 \\
\hline \multirow{6}{*}{ Tipo de enfermedad de base ${ }^{\Omega}$} & Cardiovascular & 15 & (33) & 12 & (14) & 3,14 & $(1,33-7,38)$ & 0,009 \\
\hline & Onco-hematológica & 6 & (13) & 20 & (23) & 0,54 & $(0,20-1,45)$ & 0,22 \\
\hline & Quemadura & 12 & (27) & 8 & (9) & 3,63 & $(1,37-9,59)$ & 0,009 \\
\hline & Malformación gastrointestinal & 3 & (7) & 17 & (20) & & & \\
\hline & Inmunodeficiencia & 3 & (7) & 1 & (12) & & & \\
\hline & Otras $^{\ddagger}$ & 6 & (13) & 28 & $(32)$ & & & \\
\hline \multicolumn{2}{|l|}{ Procedimiento invasor previo ${ }^{\alpha}$} & 50 & $(100)$ & 83 & (83) & & & \\
\hline \multicolumn{2}{|l|}{ Catéter venoso central } & 50 & $(100)$ & 72 & (72) & & & \\
\hline \multicolumn{2}{|l|}{ Catéter vesical } & 26 & $(52)$ & 32 & (32) & 2,9 & $(1,40-6)$ & 0,004 \\
\hline \multicolumn{2}{|c|}{ Asistencia respiratoria mecánica } & 45 & (90) & 35 & (35) & 10 & $(4,07-25)$ & 0,0001 \\
\hline \multicolumn{2}{|l|}{ Cirugía } & 31 & $(62)$ & 39 & (39) & 2,5 & $(1,26-5,1)$ & 0,009 \\
\hline \multicolumn{2}{|c|}{ Internación previa (días) (mediana-IC) } & 16( & $(6,5-32)$ & 7 & $(3-21,5)$ & & & 0,0001 \\
\hline \multicolumn{2}{|l|}{ ATB parenterales previos ${ }^{\pi}$} & 47 & (94) & 57 & $(57)$ & 11,8 & $(3,44-40)$ & 0,0001 \\
\hline \multicolumn{2}{|l|}{ Tratamiento inmunosupresor } & 10 & (20) & 30 & (30) & 0,58 & $(0,25-1,3)$ & 0,19 \\
\hline \multicolumn{2}{|l|}{ Leucopenia } & 8 & (16) & 19 & (19) & 0,81 & $(0,32-2)$ & 0,65 \\
\hline \multicolumn{2}{|l|}{ Neutropenia } & 5 & (10) & 14 & (14) & 0,68 & $(0,23-2)$ & 0,48 \\
\hline \multicolumn{2}{|l|}{ Foco clínico inicial } & 24 & $(48)$ & 39 & (38) & 1,38 & $(0,85-2,2)$ & 0,18 \\
\hline \multicolumn{2}{|l|}{ Sepsis } & 26 & $(52)$ & 52 & (52) & 1,17 & $(0,59-2,31)$ & 0,64 \\
\hline \multicolumn{2}{|l|}{ Fallecimiento } & 3 & (7) & 4 & (4) & & & \\
\hline \multicolumn{9}{|c|}{ 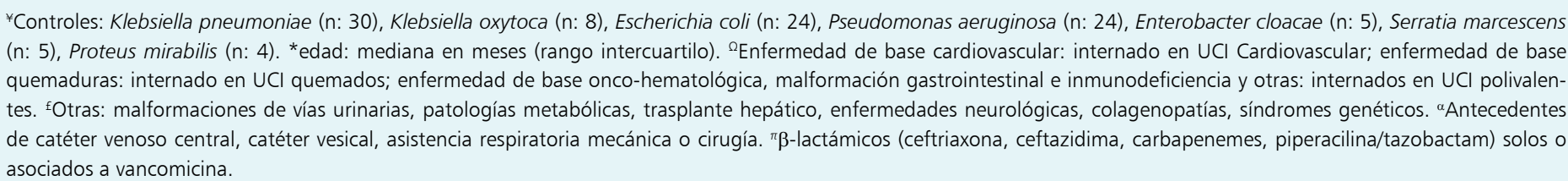 } \\
\hline
\end{tabular}

Al analizar las características de los pacientes (Tabla 1), no se observaron diferencias significativas respecto a edad, sexo, presencia de enfermedad de base, tratamiento inmunosupresor, presencia de leucopenia o neutropenia, forma de presentación clínica, presencia de sepsis ni en la evolución clínica final de las bacteriemias por ABM respecto de aquellas por otros bacilos gramnegativos. Se encontró diferencia estadísticamente significativa en la mediana de días de internación previos a la aparición de la bacteriemia por ABM, que fue de 16 días en los casos y 7 días en los controles ( $p<0,01)$. Al analizar el tipo de procedimiento invasor, todos los casos habían tenido CVC antes o lo tenían durante el episodio de bacteriemia.

Se observaron diferencias significativas respecto a la utilización de ARM (90 vs 35\%, OR 10, IC 95\% 4,07$25,0 \mathrm{p}<0,01$ ), uso de catéter vesical (52 vs $32 \%$ OR
2,9, IC 95\% 1,40-6,0 p < 0,01), antecedente de cirugía reciente (62 vs 39\% OR 2,55, IC 95\% 1,26-5,1 p <0,01) y utilización de antimicrobianos de amplio espectro previa a la aparición de la bacteriemia (94 vs $57 \%$, OR 11,81, IC 95\% 3,44-40,53,<0,01) (Tabla 1). Tuvieron mayor riesgo de presentar bacteriemia por ABM los pacientes con cardiopatía o quemadura, no observándose diferencia en niños con otras patologías subyacentes (Tabla 1).

En el grupo de los casos, la bacteriemia fue asociada a CVC en $40 \%$ (n: 20), a infección de quemadura $24 \%$ (n: 12), bacteriemia primaria en $22 \%$ (n: 11), secundaria asociada a infección de sitio quirúrgico en $10 \%$ (n: 5) y relacionada con neumonía asociada a respirador en $4 \%$ (n: 2). El tratamiento implementado fue: colistín monoterapia en 58\% (n: 29) o asociado a: carbapenemes 26\% (n: 13), a TAZ 10\% (n: 5), a AMS 4\% (n: 2) o a FEP 2\% (n: 1). La 
Tabla 2. Susceptibilidad antimicrobiana de los 50 aislados de Acinetobacter baumannii

\begin{tabular}{llcc} 
Tipo de ATB & ATB & $\begin{array}{c}\text { Difusión } \\
\text { \% resistencia }\end{array}$ & $\begin{array}{c}\text { Dilución } \\
\text { Rango de CIM }(\boldsymbol{\mu} \mathbf{g} / \mathbf{m l})\end{array}$ \\
Penicilinas & PIP & 100 & \\
$\beta$-lactámicos combinados con inhibidores & AMS & 98 & \\
& TAZ/AMC & 100 & \\
Cefalosporinas & CAZ & 98 & $8-128$ \\
Carbapenemes & FEP & 100 & $8-128$ \\
& MEM & 100 & $16-256$ \\
& MEM+EDTA & & $16-256$ \\
Lipopéptidos & IMI & 100 & \\
Aminoglucósidos & IMI+EDTA & & \\
Quinolonas & COL & 0 & \\
Inhibidor del folato & AK & 100 & \\
Tetraciclinas & GEN & 72 & \\
\hline
\end{tabular}

PIP: piperacilina, AMS: ampicilina/sulbactam, TAZ: piperacilina/tazobactam, AMC: amoxicilina/clavulánico CAZ: ceftazidima, FEP: cefepime, IMI: imipenem, MEM: meropenem, COL: colistín, AK: amikacina, GEN: gentamicina, NAL: ácido nalidíxico, CIP: ciprofloxacina, STX: cotrimoxazol, MIN: minociclina, EDTA: ácido etilendiaminotetraacético.

\section{Tabla 3. Resultados de curvas de muerte realizadas con los 50 aislados}

$\begin{array}{ccccc}\text { n de aislados } & \text { Clones } & \text { AMS } & \text { POL } & \text { AMS+GEN } \\ \text { n: } 28 & \text { I } & - & + & \text { Sin } \\ \text { n: } 10 & 2 & - & + & \text { Sin } \\ \text { n: } 3 & 3 & - & + & \text { Indif } \\ \text { n: } 6 & \text { IV } & - & + & \text { Indif } \\ \text { n: } 3 & 5 & - & + & \text { Sin }\end{array}$

+: efecto bactericida: es la disminución de $\geq 3 \log$ en ufc/ml con respecto al inóculo inicial, - tolerancia: es la disminución de $<3$ log en ufc/ml con respecto al inóculo inicial, Sin:, sinergia, es la disminución de $\geq 2$ log entre la combinación y el antimicrobiano más activo y el inóculo inicial. Indif: indiferencia, es la disminución o el aumento menor de 2 log entre la combinación y el antimicrobiano más activo y el inóculo inicial. espectro ( $\beta$-lactámicos) (OR 7 IC 95\% 2-25, p < 0,01) y el antecedente de ARM (OR 5,9, IC 95\% 2,4-14, p <0,001).

En la Tabla 2 se describen los patrones de susceptibilidad antimicrobiana observados en los 50 ABM incluidos en el estudio. La susceptibilidad a MEM e IMI se estudió comparando los métodos de difusión y dilución, y se obtuvieron iguales resultados. El agregado de EDTA 0,4 mM no modificó los valores de las CIM para los carbapenemes ensayados (Tabla 2).

En la serie de casos evaluada no se hallaron BLEE ni carbapenemasas de tipo metalo- $\beta$-lactamasas; pero sí se confirmó la presencia de carbapenemasas de tipo oxacilinasas en $94 \%$ de los aislados. Se detectó la presencia del gen oxa 23 en 44 aislados (88\%) y el gen oxa 58 en otras $3(6 \%)$.

Al analizar la clonalidad en la colección se encontraron cinco clones diferentes de $A$. baumannii (Figura 1). La polimixina $\mathrm{B}$ mostró efecto bactericida en todos los clones por el método de curvas de muerte (Tabla 3). Además, en tres de cinco clones se observó sinergia con la combinación AMS + gentamicina.

\section{Discusión}

Las infecciones por ABM constituyen uno de los principales problemas causados por patógenos emergentes en las UCI de los hospitales de alta complejidad de nuestro país. Diversos estudios fueron publicados sobre este tema y en su mayoría corresponden a series de pacientes $\operatorname{adultos}^{14,15}$.

En el presente trabajo, las patologías subyacentes de los pacientes fueron similares a las reportadas en otras series ${ }^{16,17}$. Los pacientes con mayor riesgo de adquisición de bacteriemia por ABM fueron aquellos con cardiopatías congénitas y los internados en la unidad de quemados. Esto probablemente tuvo relación con la alta frecuencia de ABM en bacteriemias, tanto en la unidad de cirugía cardiovascular como en la unidad de pacientes quemados durante el período de estudio.

Las UCI son un lugar frecuente de adquisición de $\mathrm{ABM}$; esto tiene relación con el tipo de pacientes internados, la exposición a procedimientos invasores y a esquemas antimicrobianos de amplio espectro, sumado a la posible transmisión horizontal que puede ocurrir ocasionando brotes ${ }^{16-18}$.

En el estudio presentado, coincidiendo con lo publicado en otras series, la bacteriemia por ABM fue más frecuente en pacientes con mayor tiempo de internación en $\mathrm{UCI}^{19,20}$.

Predominaron las bacteriemias relacionadas a CVC y en segunda instancia a infección de la quemadura; a diferencia de la población adulta donde el foco pulmonar constituye uno de los focos más frecuentemente reporta- 
$\operatorname{dos}^{18}$. La frecuencia de bacteriemia primaria observada en nuestro estudio fue similar a la reportada en otra serie ${ }^{19}$. El motivo de aparición de las bacteriemias primarias por ABM aún no está claro. Algunos autores proponen el origen intestinal, por traslocación bacteriana, fenómeno frecuente en pacientes críticamente enfermos ${ }^{20}$.

En el análisis de regresión logística múltiple se identificaron como predictores independientes de bacteriemia por $\mathrm{ABM}$ al antecedente de haber recibido tratamiento antimicrobiano (principalmente carbapenemes y cefalosporinas de espectro extendido) y el uso de ARM. Estos factores son similares a los reportados en otras series, en las que se resalta el impacto de la utilización de antimicrobianos de amplio espectro en las UCI respecto a la emergencia de ABM. Asimismo, se destaca la relación entre la ARM y la mayor posibilidad de colonización y posterior infección en pacientes críticos sometidos a procedimientos invasores ${ }^{21,22}$. Punpanich y cols., identificaron también el antecedente de ARM y la exposición previa a carbapenémicos como factores de riesgo independientes para la adquisición de bacteriemia por ABM en niños ${ }^{23}$.

En nuestra serie, la mayoría de los pacientes recibió monoterapia con colistín y presentaron buena evolución clínica. El tratamiento más eficaz para las infecciones por $\mathrm{ABM}^{24}$ y las ventajas del tratamiento combinado ${ }^{25,26}$ están todavía en discusión. Algunos autores sugieren utilizar ampicilina/sulbactam en combinación con polimixinas ${ }^{27}$. Un estudio multicéntrico que comparó el tratamiento del ABM con colistín monoterapia vs colistín en combinación con rifampicina no encontró diferencias en mortalidad ni en duración de la internación entre ambos grupos ${ }^{28}$. Coincidente con lo descrito en otros estudios sobre el uso de colistín en pediatría, la frecuencia de toxicidad renal fue baja ${ }^{29,30}$.

En el estudio presentado no se identificaron aislados de ABM resistentes a colistín; sin embargo, en estudios recientemente publicados se describe $11 \%$ de resistencia a colistín en pacientes con infecciones por A. baumannii en $\mathrm{UCI}^{31}$.

La mortalidad reportada en la literatura científica para infecciones por ABM en población adulta es variable ${ }^{32,33}$. $\mathrm{Hu}$ y cols., realizaron una revisión sistemática de las infecciones invasoras por ABM, encontrando una mortalidad de $14,5 \%{ }^{34}$.

Algunos autores han encontrado asociación entre la evolución de las infecciones por ABM y la gravedad de los pacientes en el momento de la infección objetivada mediante scores de gravedad ${ }^{35}$. Esto no pudo ser evaluado en el presente estudio, por su carácter retrospectivo y no contar con la categorización de gravedad al ingreso de los pacientes incluidos según scores.
En el estudio de clonalidad, se encontraron cinco clones diferentes de ABM en el período evaluado. Otros autores han descrito la circulación simultánea de distintos clones y la heterogeneidad en las cepas de ABM en el ámbito intrahospitalario ${ }^{36}$.

Las limitaciones del estudio radican en su naturaleza retrospectiva y la imposibilidad de establecer la categorización de gravedad de los pacientes al momento de la aparición de la bacteriemia.

Es necesario implementar programas de prevención y de uso de antimicrobianos en UCI para el control de las infecciones por $\mathrm{ABM}$.

\section{Conclusiones}

Las bacteriemias por ABM en niños internados en UCI se observaron en pacientes con patología subyacente, internación prolongada, sometidos a procedimientos invasores y a tratamiento antimicrobiano de amplio espectro.

\section{Resumen}

Introducción: Acinetobacter baumannii multi-resistente $(\mathrm{ABM})$ es un patógeno intrahospitalario de importancia. Objetivos: Analizar factores de riesgo de adquisición y características clínicas y microbiológicas de las bacteriemias por ABM (BABM) en pediatría. Métodos: Estudio de casos y controles período 2005-2008. Se incluyeron variables demográficas y clínicas de pacientes con BABM y por otros bacilos gramnegativos no ABM. Se realizaron pruebas para identificación de especie, susceptibilidad antimicrobiana y detección feno-genotípica de mecanismos de resistencia, sinergia y clonalidad. Análisis estadístico: Stata 8.0. Resultados: Se incluyeron 50 BABM y 100 controles. El 94\% de los pacientes adquirieron la BABM en UCI y $88 \%$ tenía patologías subyacentes. La mediana de días de internación previa a la bacteriemia fue mayor en los casos ( 16 vs 7 días, $\mathrm{p}<0,001)$. Se detectaron cinco clones de ABM. Se encontró efecto bactericida in vitro con polimixina B y con ampicilina/sulbactam+gentamicina. Tres casos fallecieron. Análisis multivariado: predictores finales de BABM fueron: antimicrobiano previo de amplio espectro [OR: 7,0; IC 95\% 1,93-25,0; p: 0,003] y asistencia respiratoria mecánica (ARM) [OR: 4,19; IC 95\% 1,66-10,0; p: 0,002]. Conclusiones: Las BABM fueron detectadas en pacientes con enfermedad subyacente, con procedimientos invasores previos e internación prolongada. Fueron predictores de BABM el tratamiento antimicrobiano de amplio espectro y ARM previa. 


\section{Referencias bibliográficas}

1.- Dijkshoorn L, Nemec A, Seifert H. An increasing threat in hospitals: multidrugresistant Acinetobacter baumannii. Nat Rev Microbiol 2007; 5: 939-51.

2.- Muñoz-Price L, Weinstein R. Acinetobacter infection. N Engl J Med 2008; 358: 1271-81.

3.- Al-Hasan M N, Huskins W C, Lahr B D, Eckel-Passow J E, Baddour L M. Epidemiology and outcome of gram-negative bloodstream infection in children: a population-based study. Epidemiol Infect 2011; 139: 791-6.

4.- Horan T C, Andrus M, Dudeck M A. CDC/ NHSN surveillance definition of health-care associated infection and criteria for specific types of infections in the acute care setting. Am J Infect Control 2008; 36: 309-32.

5 .- Bouvet A, Grimont P A. Identification and biotyping of clinical isolates of Acinetobacter. Ann Inst Pasteur Microbiol 1987; 138: 569-78.

6.- Brown S, Young H, Amyes S. Characterisation of OXA-51, a novel class D carbapenemase found in genetically unrelated clinical strains of Acinetobacter baumannii from Argentina. Clin Microbiol Infect 2005; 11: 15-23.

7.- Clinical and Laboratory Standards Institute (CLSI) Performance standards for antimicrobial susceptibility testing; 19th informational supplement M100-S19. Clinical and Laboratory Standards Institute Wayne, Pa. 2009.

8.- National Committee for Clinical Laboratory Standards. Performance standards for antimicrobial disk susceptibility test, 8 th ed. Approved Standard M2-A8. National Committee for Clinical Laboratory Standards, Wayne, Pa, 2003

9.- Lee K, Lim Y, Yong D, Yum J H, Chong Y. Evaluation of the Hodge test and the imipenem-EDTA double-disk synergy test for differentiating metallo- $\beta$-lactamase-producing isolates of Pseudomonas spp. and Acinetobacter spp. J Clin Microbiol 2003; 41: 4623-9.

10 .- Pasterán F, Rapoport M, Petroni A, Faccone D, Corso A, Galas M, et al. Emergence of PER-2 and VEB-1a in Acinetobacter baumannii strains in the Americas Antimicrob Agents Chemother 2006; 50: 3222-4.

11 .- Limansky A, Viale A. Can composition and structural features of oligonucleotides contribute to their wide-scale applicability as random PCR primers in mapping bacterial genome diversity? J Microbiol Methods 2002; 50: 291-7.

12 .- Lorian V. (ed.) Antibiotics in Laboratory Medicine. 4th ed. Williams \& Wilkins, Baltimore, USA, 1996.

13.- Landman D, Georgescu C, Martin D A, Quale J. Polymyxins revisited. Clin Microbiol Rev 2008; 21: 449-65.

14 .- Tseng Y C, Wang J T, Wu F L, Chen Y C,
Chie W C, Chang S C. Prognosis of adult patients with bacteremia caused by extensively resistant Acinetobacter baumannii. Diagn Microbiol Infect Dis 2007; 59: 181-90.

15 .- Playford E G, Craig J C, Iredell J R. Carbapenem-resistant Acinetobacter baumannii in intensive care unit patients: risk factors for acquisition, infection and their consequences. J Hosp Infect 2007; 65: 204-11.

16.- Kuo L C, Y u C J, Lee L N, Wang J L, Wang $\mathrm{H} \mathrm{C}$, Hsueh P R, et al. Clinical features of pandrug-resistant Acinetobacter baumannii bacteremia at a university hospital in Taiwan. J Formos Med Assoc 2003; 102: 601-6.

17.- Esterly J, Griffith M, Qi Ch, Malczynski M, Postelnick M J, Scheetz M H, et al. Impact of carbapenem resistance and receipt of active antimicrobial therapy on clinical outcomes of Acinetobacter baumannii bloodstream infections. Antimicrob Agents Chemother 2011; 55: 4844-9.

18.- Tognim M C, Andrade S S, Silbert S, Gales A C, Jones R N, Sader H S. Resistance trends of Acinetobacter spp. in Latin America and characterization of international dissemination of multi-drug resistant strains: five-year report of the SENTRY Antimicrobial Surveillance Program. Int J Infect Dis 2004; 8: 284-91.

19.- Rattanaumpawan P, Ussavasodhi P, Kiratisin P, Aswapokee N. Epidemiology of bacteremia caused by uncommon non-fermentative gramnegative bacteria. BMC Infect Dis 2013; 13: 167.

20 -- Cisneros J, Rodríguez-Baño J. Nosocomial bacteremia due to Acinetobacter baumannii: epidemiology, clinical features and treatment. Clin Microbiol Infect 2002; 8: 687-93.

21 .- Jung J Y, Park M S, Kim S E, Park B H, Son J Y, Kim E Y, et al. Risk factors for multi-drug resistant Acinetobacter baumannii bacteremia in patients with colonization in the intensive care unit. BMC Infect Dis 2010; 10: 228.

22 .- Sheng $W H$, Liao $C H$, Lauderdale $T$ L, Ko W C, Chen Y S, Liu J W, et al. A multicenter study of risk factors and outcome of hospitalized patients with infections due to carbapenemresistant Acinetobacter baumannii. Int J Infect Dis 2010; 14: e764-9.

23 .- Punpanich W, Nithitamsakun N, Treeratweeraphong V, Suntarattiwong P. Risk factors for carbapenem non-susceptibility and mortality in Acinetobacter baumannii bacteremia in children. Int J Infect Dis 2012; 16: e811-5.

24 .- Michalopoulos A, Falagas M E. Treatment of Acinetobacter infections. Exp Opin Pharmacother 2010; 11: 779-88.

25 .- Karaoglan I, Zer Y, Bosnak VK, Mete AO,
Mete AO, Namiduru M. In vitro synergistic activity of colistin with tigecycline or $\beta$-lactam antibiotic/ $\beta$-lactamase inhibitor combinations against carbapenem-resistant Acinetobacter baumannii. J Int Med Res 2013; 41: 1830-7.

26.- Wang X, Qiao F, Yu R, Gao Y, Yong Z. Clonal diversity of Acinetobacter baumannii clinical isolates revealed by a snapshot study. BMC Microbiol 2013; 13: 234.

27 .- Hsu A J, Tamma P D. Treatment of multidrugresistant gram-negative infections in children. Clin Infect Dis 2014; 58 (10): 1439-48.

28.- Durante-Mangoni E, Signoriello G, Andini R, Mattei A, De Cristoforo M, Murino P, et al. Colistin and rifampicin compared with colistin alone for the treatment of serious infections due to extensively drug-resistant Acinetobacter baumannii:a multicenter, randomized clinical trial. Clin Infect Dis 2013; 57: 349-58.

29.- Rosanova M, Epelbaum C, Noman A, Villasboas M, Álvarez V, Berberian G, et al. Use of colistin in a pediatric burn unit in Argentina. J Burn Care Res 2009; 30: 612-5.

30.- Falagas M, Sideri G, Vouloumanou E, Papadatos J, Kafetzis D A. Intravenous colistimethate (colistin) use in critically ill children without cystic fibrosis Pediatr Infect Dis J 2009; 28: 123-7.

31 .- Vakili B, Fazeli H, Shoaei P, Yaran M, Ataei B, Khorvash F, et al. Detection of colistin sensitivity in clinical isolates of Acinetobacter baumannii in Iran. J Res Med Sci 2014; 19: S67-70.

32 .- Mathai A S, Oberoi A, Madhavan S, Kaur P. Acinetobacter infections in a tertiary level intensive care unit in northern India: Epidemiology, clinical profiles and outcomes. J Infect Public Health 2012; 5: 145-2.

33 .- Lemos E, De la Hoz F, Restrepo N, Alvis N, Cañon O, León Y. Mortalidad por Acinetobacter baumannii en unidades de cuidados intensivos en Colombia. Rev Panam Salud Publica 2011; 30: 287-94.

34.- Hu J, Robinson J L. Systematic review of invasive Acinetobacter infections in children. Can J Infect Dis Med Microbiol 2010; 21: 83-8.

35.- Chen S J, Chao T F, Chiang M C, Kuo S C, Chen L Y, Yin T, et al. Prediction of patient outcome from Acinetobacter baumannii bacteremia with Sequential Organ Failure Assessment (SOFA) and Acute Physiology and Chronic Health Evaluation (APACHE) II scores Intern Med 2011; 50: 871-7.

36.- Minandri F, D’Arezzo S, Antunes L C, Pourcel C, Principe L, Petrosillo N, et al. Evidence of diversity among epidemiologically related carbapenemase-producing Acinetobacter baumannii strains belonging to international clonal lineage II. J Clin Microbiol 2012; 50: 590-7. 